\title{
The COVID-19 Epidemic: Management and Outcomes of Hemodialysis and Peritoneal Dialysis Patients in Stockholm, Sweden
}

\author{
Jessica Smolander ${ }^{a} \quad$ Annette Bruchfeld ${ }^{b, c}$ \\ aDepartment of Renal Medicine, Danderyd Hospital, Stockholm, Sweden; bepartment of Health, Medicine and \\ Caring Sciences, Linköping University, Linköping, Sweden; 'Department of Renal Medicine, Karolinska University \\ Hospital and CLINTEC KI, Stockholm, Sweden
}

\section{Keywords}

COVID-19 · Hemodialysis and peritoneal dialysis .

Management · Outcome · Chronic kidney disease

\begin{abstract}
Background: The COVID-19 outbreak has been associated with a high morbidity, mortality, and a risk of long-term sequelae, and patients with severe COVID-19 are at increased risk of acute kidney injury. CKD patients are at high risk of being exposed to COVID-19 and suffer complications and poor outcome. In Sweden, mitigation strategies did not include lockdown. During March-April of 2020, wide-spread infection occurred in Stockholm. Methods: Management and outcomes in forty hemodialysis (HD) patients and 4 peritoneal dialysis (PD) patients, with symptomatic COVID-19 in greater Stockholm during March and April of 2020 are reported. $R$ esults: Twenty-four HD patients (60\%) required medical care and hospitalization, whereas 16 patients (40\%) were treated at home. Nine patients died (mortality rate of $22.5 \%$ ), of whom 8 were men. The median age in non-survivors (78 years) was significantly higher than in survivors $(p=0.003)$. The median time in dialysis (11.5 years) was also significantly longer in non-survivors ( $p=0.01)$. C-reactive protein (CRP) at diagnosis in 7 of non-survivors (median 213 mg/L, range 86$329 \mathrm{mg} / \mathrm{L}$ ) was significantly higher than the CRP in 25 survi-
\end{abstract}

karger@karger.com www.karger.com/kbr

Karger $\stackrel{\text { ' }}{5}$

GOPEN ACCESS
(C) 2021 The Author(s)

Published by S. Karger AG, Basel

This is an Open Access article licensed under the Creative Commons Attribution-NonCommercial-4.0 International License (CC BY-NC) (http://www.karger.com/Services/OpenAccessLicense), applicable to the online version of the article only. Usage and distribution for commercial purposes requires written permission. vors (median $87 \mathrm{mg} / \mathrm{L}$, range $1-328 \mathrm{mg} / \mathrm{L})(p=0.0003)$. Maximum CRP also indicated poorer outcome among hospitalized patients $(p=0.0004)$. The gender imbalance was striking with only men dying apart from 1 elderly woman. Only 4 PD patients were hospitalized with symptomatic COVID-19. One patient died, 2 were discharged, and 1 was treated at the intensive care unit and survived. Conclusion: HD patients $>70$ years were reported with longer dialysis vintage, higher CRP, and males were at an increased risk of dying from COVID-19, whereas those $<70$ years seemed to have a milder disease. Mitigation strategies to reduce rates of infection in high-risk populations remain essential. Follow-up focusing on longterm prognosis for extrapulmonary manifestations is likely to be important also in dialysis patients.

(C) 2021 The Author(s)

Published by S. Karger AG, Basel

\section{Introduction}

Severe acute respiratory syndrome-corona virus (SARS-CoV)-2 was first described in China in December 2019, and the COVID-19 outbreak was later declared as a pandemic by the WHO. As of November 26, 2020, close to 60 million individuals have been infected and 1.4 million deaths have been reported. The most frequent disease manifestation is fever with viral pneumonia, which 
in more severe cases requires hospital care and may lead to respiratory failure and need of mechanical ventilation. Hyperinflammation, multiorgan failure, and coagulation disorder all contribute to a high mortality $[1,2]$. Early data from China and Italy indicated that higher age, male sex, and comorbidities such as cardiovascular disease, diabetes, and CKD are risk factors for severe illness and death. However, the severity of underlying conditions may vary between individuals. Hence, most of these patients will not develop critical illness and require intensive care resources or even hospital care [3,4]. Obesity has also been highlighted as a risk factor, but in a New York study of over 5,000 COVID-19-infected individuals, age and underlying CKD were stronger predictors of severe disease than obesity [5]. The largest risk-factor study to date is from the United Kingdom which included 17 million electronic health records covering $40 \%$ of the population. The data set identified CKD as a risk factor for mortality in patients with COVID-19, in particular in those with a glomerular filtration rate $<30 \mathrm{~mL} / \mathrm{min} / 1.73 \mathrm{~m}^{2}$ and organ transplantation which both conferred a high risk in multivariate analyses. This study also highlighted increased risks associated with deprivation [6].

Although lung involvement is the most common and most serious disease manifestation, it has been increasingly demonstrated that extrapulmonary manifestations are quite common [7]. The kidneys can be affected in several ways, and acute kidney injury (AKI) is relatively common as well as hematuria and proteinuria. From the USA and the UK, AKI figures of $20-46 \%$ have been reported, which in the intensive care unit (ICU) setting in combination with the need for kidney replacement therapy (KRT) also dramatically increases the risk of mortality $[8,9]$.

It is not yet known whether early signs of renal damage in COVID-19 will increase the risk of CKD in the long term. Still, the striking clinical presentation of hematuria and/or proteinuria as well as AKI and the negative impact on outcome adds further evidence to kidneys being an additional target organ for SARS-CoV-2.

Nephrologists have long been aware of links between viral infections and immune-mediated kidney disease [10]. One well-known example is hepatitis $C$, which is associated with glomerulonephritis and vasculitis leading to an increased risk of CKD [11]. Interestingly, reports of collapsing glomerulopathy in COVID-19-associated AKI have now been published, resembling what has previously been described in HIV [12]. Last, a very high risk of thrombotic complications is particularly prominent in COVID-19, suggesting that the endothelium also is a target organ $[13,14]$.

COVID-19 in Hemodialysis and Peritoneal Dialysis Patients in Sweden
In this article, we describe the COVID-19 epidemic, management, and outcomes in the dialysis population of Stockholm, the part of Sweden with the highest infection rates in March-April of 2020. We also present 2 clinical dialysis patient case reports who were admitted to the ICU.

\section{Material and Methods}

\section{Symptomatic COVID-19 in Hemodialysis Patients and \\ Outcome}

The Stockholm region has been the epicenter of the COVID-19 epidemic in Sweden until recently. The mortality rate in the Stockholm region reached approximately 100 per 100,000 inhabitants by the end of July 2020 as reported by the Public Health Agency of Sweden [15]. No lockdown was put in place, rather a series of recommendations including social distancing, reducing the maximum number of people at gatherings, and hand hygiene were implemented. Working from home was suggested if possible and virtual lessons for university and high-school students were started.

At the beginning of March of 2020, there were 520 hemodialysis (HD) patients in the greater Stockholm area. HD patients were tested for SARS-CoV-2 with PCR if they presented with fever, respiratory, or gastrointestinal symptoms. If confirmed, positive patients were to follow recommendations from the Public Health Agency, and moreover be isolated during their dialysis session. Dialysis sessions were rapidly rescheduled to create cohorts where infected patients were dialyzed together to protect noninfected patients. No mandatory wearing of facemasks was introduced. SARSCoV-2-positive patients were considered free of infection if they were symptom-free for $72 \mathrm{~h}$ and at least 7 days had passed from first symptoms. During the period from March 12 to April 17, 2020, a total of forty symptomatic patients were tested positive for SARS-CoV-2 (see Table 1). Out of these 40 infected patients, 24 patients had COVID-19 requiring further medical care. Twentytwo patients were hospitalized, 1 died of cardiac arrest in the emergency room, and another received palliative care at a nursing home. Nine patients with more severe COVID-19 infection died (mortality rate of $22.5 \%$ ), of whom 8 were men and older than 70 years. The median age in the deceased group (78 years) was significantly higher than those who survived (Student's t-test; $p=$ $0.003)$, and the median time in dialysis ( 11.5 years) was significantly longer than that of the survivors $(p=0.01)$. One of the nonsurvivors was hospitalized and subsequently treated with mechanical ventilation in the ICU (see Case 1). In the remaining cases, a decision was made not to admit to the ICU because of the patient's wishes, age, or comorbidities. The only woman who died was 96 years old and had undergone dialysis for a total of 19 years.

Out of the 15 hospitalized and surviving HD patients, $60 \%$ were treated with oxygen, but needed neither high-flow oxygen treatment nor admission to the ICU. Most patients were given antibiotics for suspected bacterial superinfections. Other than the HD patient admitted to the ICU, none were given antimalarial drugs or anti-inflammatory therapy. In the hospitalized survivors, $87 \%$ were men, the median age was lower (68 years), and the median time in dialysis was significantly shorter ( 1.5 years) than in nonsurvivors. However, the 2 women in this group had a significantly 
Table 1. Symptomatic COVID-19 infection in the Stockholm HD population, March 12 to April 17, 2020

\begin{tabular}{lccc}
\hline Variables & $\begin{array}{l}\text { Hospitalized } \\
\text { non-survivors }\end{array}$ & $\begin{array}{l}\text { Hospitalized } \\
\text { survivors }\end{array}$ & Outpatient \\
\hline Patients, $n(\%)$ & $9(22.5)$ & $15(37.5)$ & $16(40)$ \\
Male, $n(\%)$ & $8(89)$ & $13(87)$ & $10(63)$ \\
Median age, yr (range) & $78(62-96)$ & $68(57-85)$ & $55.5(19-86)$ \\
Age $\geq 70, n(\%)$ & $8(89)$ & $7(47)$ & $4(25)$ \\
Median dialysis vintage, yr (range) & $11.5(6-17$ y) & $1.5(1$ mo-19 y) & $3(1$ mo-12 y) \\
Oxygen dependent, $n(\%)$ & $9(100)$ & $9(60)$ & $0(0)$ \\
ICU, $n$ (\%) & $1(11)$ & $0(0)$ & na \\
Diabetes mellitus, $n(\%)$ & $5(56)$ & $8(53)$ & $9(56)$ \\
BMI $>30$ kg/m ${ }^{2}, n(\%)$ & $0(0)$ & $2(13)$ & $9(6)$ \\
RAAS-blockade, $n(\%)$ & $2(22)$ & $1(40)$ & $4(25)$ \\
Immunosuppressive therapy, $n(\%)$ & $0(0)$ & & \\
\hline
\end{tabular}

Patients $n=40$. HD, hemodialysis; ICU, intensive care unit.

longer dialysis vintage (14 and 19 years, respectively). Among the 16 patients who were followed up in an outpatient setting, 63\% were men, with an even lower median age ( 55.5 years), but with a greater age distribution (19-86 years). Median time in dialysis was 3 years. This descriptive data are summarized in Table 1.

Plasma C-reactive protein (CRP) at diagnosis was available in 7 of the non-survivors (median $213 \mathrm{mg} / \mathrm{L}$, range $86-329 \mathrm{mg} / \mathrm{L}$, reference $<3 \mathrm{mg} / \mathrm{L}$ ) and in 25 of the survivors (median $87 \mathrm{mg} / \mathrm{L}$, range $1-328 \mathrm{mg} / \mathrm{L}$ ) and was significantly higher in the non-survivors $(p=0.0003)$. Maximum CRP also indicated poorer outcomes among hospitalized patients $(p=0.0004)$. Lymphocyte count was available at diagnosis in 22 patients but did not differ significantly between survivors and non-survivors $(p=0.28)$.

The proportion of patients with diabetes did not differ between groups (53-56\%). Only 3 of the HD patients had a BMI $>30 \mathrm{~kg} / \mathrm{m}^{2}$, and 5 patients were on immunosuppressive therapy, but none of these factors increased the risk of more serious disease in this small group. There were no differences between the groups regarding hypertension or prior cardiovascular disease. Among survivors, the proportion of patients with ongoing treatment with either an ACE inhibitor or ARB was not significantly different from nonsurvivors $(p=0.08)$. Thirteen out of 15 patients continued their treatment during the infection without developing more serious disease.

Universal masking was introduced for all in-center HD patients traveling from their homes in early April. The dialysis staff were also better equipped with personal protection equipment. From May through August, only sporadic cases have been diagnosed.

\section{Peritoneal Dialysis Patients with COVID-19 Requiring \\ Hospitalization and Outcome}

Patients with peritoneal dialysis (PD) manage their dialysis at home and were only tested if hospitalized for COVID-19. In the Stockholm region, there were $127 \mathrm{PD}$ patients at the beginning of the epidemic. Only 4 of these patients were hospitalized by April 17 due to COVID-19. Out of these, a 73-year-old patient died. Another 54-year-old was admitted to the ICU and survived (see Case 2 ). The other 2 were 42 and 56 years old, respectively, needed oxy- gen, and were hospitalized for 8-9 days. The median time in dialysis for these 4 patients was 2 years. Two had diabetes and all had ongoing treatment with an ACE inhibitor or ARB.

\section{Case Reports}

Case 1: The patient was a 62-year-old man with end-stage kidney disease (ESKD) due to polycystic kidney disease. He previously had a kidney transplant but was currently on home HD since 17 years. He presented with GI symptoms, was hospitalized on March 20, and tested positive for SARS-CoV-2. A decision of no admission to the ICU due to ESKD with dialysis treatment was made but was retracted after discussion with the nephrology team. Due to worsening pneumonia, the patient was intubated on March 23. He was however hemodynamically unstable and increasingly difficult to ventilate. The patient did not respond well to proning or high doses of vasopressors. The fever was high and CRP was $496 \mathrm{mg} / \mathrm{L}$ ( ref <3 mg/L), which had been rising rapidly, despite intravenous (IV) cefotaxime. Ferritin was also increasing to $918 \mu \mathrm{g} / \mathrm{L}$ (ref $30-350 \mu \mathrm{g} / \mathrm{L}$ ), as well as interleukin-6 (IL-6) which was $1,852 \mathrm{ng} / \mathrm{L}$ (ref $<7 \mathrm{ng} / \mathrm{L}$ ). Lymphopenia was mild. The patient was believed to be suffering from hyper-inflammation due to the COVID-19 infection and was given a single dose of the IL-6 inhibitor RoActemra (tocilizumab) $8 \mathrm{mg} / \mathrm{kg}$ on March 25 and also received a reduced dose of chloroquine phosphate on March 24-28. The following day, the patient was improving regarding ventilation, was afebrile, and inflammatory parameters decreased. On March 30, the patient was extubated and transferred to an intermediate care ward for respiratory oxygen support. On April 2, he developed an enterococcal sepsis which he succumbed to on April 8.

Case 2: This patient was a 54-year-old woman with ESKD likely due to hypertension. She had been on PD for 2 years waiting for a kidney transplant. She presented with 10 days of fever, fatigue, muscle aches, and vomiting. SARS-CoV-2 was positive during hospitalization, on March 24. An initial decision of no admission to the ICU due to ESKD with dialysis treatment was made but was changed after discussion with the nephrology team. The patient was subsequently transferred to the ICU and intubated on March 25 due to rapidly progressive respiratory failure. Plasma levels of 
CRP, with a maximum of $448 \mathrm{mg} / \mathrm{L}$ ( $\mathrm{ref}<3 \mathrm{mg} / \mathrm{L}$ ) had rapidly increased, as well as those of ferritin, IL-6, and fibrin-D-dimer. Lymphopenia was mild. IV cefotaxime was initiated. The patient improved and was extubated after 13 days and was subsequently transferred to an intermediate care ward and eventually to the nephrology ward with normal oxygen saturation. Sequelae such as critical illness neuropathy and cognitive symptoms were, however, prominent. The patient was sent to a rehabilitation facility but returned home 2 months after being hospitalized. Interestingly, PD treatment was continued throughout the hospitalization, also in the ICU, using manual exchanges $4-5$ times a day.

\section{Discussion}

The COVID-19 pandemic has proven to be a new challenge for nephrology. We have here presented initial descriptive data on dialysis patients in Stockholm, who were affected early and severely by the COVID-19 epidemic in Sweden. HD patients older than 70 years were at a higher risk of dying from symptomatic COVID-19, whereas those younger than 70 years seemed to have a milder disease in most cases. To the best of our knowledge, we are the first to describe that longer dialysis vintage and not only age is associated with poor outcome. This likely reflects a higher degree of frailty, which is also associated with an increased mortality from COVID-19. Interestingly, the gender balance was also skewed as very few women died in our COVID-19 cohort. Higher and maximum CRP at admission were predictive of poor outcomes. Few PD patients were hospitalized with COVID-19 infection. This is likely due to better isolation options having a home dialysis treatment than going to an in-center HD unit several times per week and thereby risking exposure. The use of good hand hygiene in $\mathrm{PD}$ may also be an advantage.

Early reports from Italy pointed to KRT patients being at high risk of infection and mortality [16]. In April, Spain reported registry data for 868 patients in KRT with COVID-19 [17], of which $63 \%$ were in $\mathrm{HD}, 4 \%$ in $\mathrm{PD}$, and $36 \%$ had a kidney transplant. A total of $85 \%$ were hospitalized, of which $8 \%$ were admitted to the ICU. Mortality reached $23 \%$ and significant risk factors for death were age and pneumonia. In Spain, many patients received hydroxychloroquine as reports initially had suggested that there could be a protective effect in COVID-19 [18]. However, randomized controlled trials (RCTs) have not shown any clinical benefit for hydroxychloroquine neither in hospitalized patients nor for early, mild COVID-19 [19]. A study from France reported 38 SARSCoV-2-infected patients out of 200 maintenance HD pa-

COVID-19 in Hemodialysis and Peritoneal Dialysis Patients in Sweden tients, with a mortality of $21 \%$. All infected patients had lymphopenia and an increase in CRP levels; however, any predictive factors regarding outcome were not presented [17]. The ERA-EDTA is currently collecting data in the newly established ERA-EDTA COVID-19 Database of Kidney Replacement Therapy patients (ERACODA) registry. Preliminary mortality data presented at the fully virtual ERA-EDTA congress in June 2020 were $25 \%$ in dialysis patients and $21 \%$ in kidney transplant patients, driven mainly by age and frailty [20]. The first published report from ERA-CODA conveyed that in 3,285 dialysis patients, among them 125 treated with $\mathrm{PD}$, the 28-day mortality risk was 21.1 times higher than the expected $1.2 \%$ mortality of propensity-score matched historic controls. Our study included far fewer patients which is a limitation, but similarly to our data, age was a predictive mortality factor and men had lower survival rates [21]. Data from PD are still rather scare but in study from a New York health system of 11 patients on chronic PD therapy, 3 patients required mechanical ventilation, and 2 died [22]. Interestingly, the mortality rate in a study from China was only 6,5\% in an HD cohort [23]. This suggests that factors other than CKD may influence outcome in various populations.

Although several of the dialysis staff and physicians were infected during the height of the epidemic, we did not collect this information. Another drawback is that not all dialysis patients, irrespective of symptoms, were tested. It has recently been proposed that a substantial number of HD patients may have asymptomatic COVID-19 infection. This was studied in London, UK, in 356 adult patients with in-center HD of whom 121 had been symptomatic and screened with a PCR test. A seroprevalence rate of $36 \%$ was found, and the study also showed that $40 \%$ of patients with SARS-CoV-2 antibodies had either asymptomatic infection or undetected disease by PCR alone [24].

During the early phase of the epidemic, there were discussions, mainly in Stockholm, on who would be likely to benefit from intensive care if critically ill and how to best determine which patients on KRT should be admitted. Decisions about the level of care are generally made after individual assessment, but should, whenever possible, also be discussed with the patient's nephrologist, the patient, and relatives. Nephrologists have expertise in assessing functional status, predicting prognosis, and prescribing treatment in patients with $\mathrm{CKD}$, dialysis, or a kidney transplant, whereas intensive-care physicians have extensive experience in treatment and treatment outcomes in the ICU and an overview of existing resourc- 
es. Previous experience in sepsis before the COVID-19 pandemic has demonstrated that HD patients are at risk of higher ICU mortality, but typically have better outcome than patients in the ICU with dialysis-dependent AKI, suggesting that ICU prognosis may be influenced by factors other than the underlying renal function [25]. As COVID-19 is a new infection, it may thus be premature to establish that dialysis patients have a considerably worse prognosis in the ICU setting than other critically ill patient groups.

The role of RAAS blockade in COVID-19 has been debated widely. Recent evidence suggests that continuing RAAS-inhibition treatment is not harmful $[26,27]$. It may, however, be too early to establish if this class of drugs has protective properties as suggested in a retrospective study from the UK and in a report from France $[28,29]$. Most of the dialysis patients in our cohort did not discontinue RAAS blockade.

The Swedish Renal Registry reported in 2019 an annual mortality in dialysis as high as $19.2 \%$, compared to only $3 \%$ in kidney transplanted patients [30]. However, dialysis patients are a heterogeneous group in terms of age, comorbidities, time in dialysis, and prognosis. A substantial group is younger, otherwise healthy, active, and working. A sizeable proportion of dialysis patients younger than 70 years and a smaller proportion in the age-group of 70-75 years are on the transplant waiting list or have a potential living donor. They are regularly assessed to ensure that they remain in a good condition to undergo transplantation, that is, general anesthesia, surgery, and complications, and thus have a good rehabilitation potential and a relatively long-life expectancy. Other dialysis patients cannot be transplanted for various reasons but are still in good physical condition and potential candidates for active treatment of COVID-19, including intensive care. However, many dialysis patients are elderly and frail with multiple comorbidities contributing to a high mortality, and we typically strive to document treatment limitation plans with these patients.

While waiting for effective COVID-19 drugs, the nephrology team should be consulted when signs of kidney involvement with hematuria and proteinuria are verified and kidney impairment is developing. The potential role of anti-inflammatory drugs in the hyperinflammatory phase of COVID-19 is increasingly recognized, although RCTs have until recently been lacking. In a smaller study from France, the risk to be admitted to the ICU was reduced for patients treated with the IL6-inhibitor tocilizumab as compared to untreated ones ( 25 vs. $72 \%$; $p=$ 0.002) [31]. In an American study, a short course of meth- ylprednisolone in 132 patients reduced the composite outcome measure ICU, mechanical ventilation, and death as compared with 81 untreated patients ( $34,9 \%$ vs. $54,3 \%$ $p=0.005)$ [32]. Recently, the randomized RECOVERY trial demonstrated that dexamethasone reduced mortality in hospitalized patients with severe respiratory complications of COVID-19 [33]. Unpublished data from the RECOVERY trial have also found a protective effect for AKI needing KRT (unpublished data, personal communication $\mathrm{R}$ Haynes). Whether these therapies may translate into better outcome also in chronic dialysis patients will be important to establish.

A recent report from Germany showed ongoing myocardial inflammation and cardiac involvement in a substantial number of recovering patients [34]. It has also been reported that approximately $10 \%$ of people experience prolonged illness after COVID-19 with symptoms ranging from serious sequelae (such as thromboembolic complications) and those with a nonspecific clinical picture, often dominated by fatigue and breathlessness [35]. It may thus be useful to assess long-term health and organ function post-COVID-19 in dialysis patients especially in those waiting for kidney transplantation.

CKD patients are, however, often excluded from RCTs, which has also been the case during the current COVID-19 pandemic [36]. One example is the antiviral drug remdesivir, which is contraindicated with a glomerular filtration rate $<30 \mathrm{~mL} / \mathrm{min}$, suggesting more pragmatic treatment strategies in the CKD population [37]. Another feature of the current epidemic is the risks of exposure for in-center HD patients suggesting that home-dialysis options should be pursued further.

AKI in severe COVID-19 has led to a surge in the need for RRT and increased mortality. The widespread clinical challenge of mobilizing not only mechanical ventilators but also all possible dialysis resources, which may compete with resources for chronic dialysis patients, has been a formidable undertaking during the first waves of this epidemic $[3-6,16,17,20-23]$. As new waves of COVID-19 emerge, mitigation policies to minimize the risk of exposing high-risk populations to the virus remain important.

\section{Conclusion}

CKD and dialysis patients are at high risk of COVID-19 infection and poor outcome. Mitigation strategies to reduce rates of infection in this population remain essential. Whether COVID-19 will increase the risk of 
CKD long-term and increase the demand for maintenance dialysis needs to be observed and investigated further. The cumulative reports of long-standing post-infectious symptoms and lingering organ damage after $\mathrm{CO}$ VID-19 suggest that this will be important to monitor also in the dialysis population.

\section{Acknowledgement}

We thank the Swedish Renal Registry for collaboration.

\section{Statement of Ethics}

The paper is exempt from Ethical Committee approval. According to Swedish law, patient data included in Quality Registries can be used for research. Patients are informed and have a right to decline to be registered, but no additional consent is required for specific projects. All patients included in this report are registered in the Swedish Renal Registry and thus no further ethical approval is required. Written informed consent was obtained from the patient and next of kin for publication of the case reports.

\section{Conflict of Interest Statement}

Jessica Smolander declares no conflict of interest. Annette Bruchfeld reports grants and personal fees from Astra Zeneca, personal fees from ChemoCentryx, Merck/MSD, and from Abbvie outside the submitted work.

\section{Funding Sources}

There was no funding for this study.

\section{Author Contributions}

The 2 authors have contributed equally to this paper.

\section{References}

1 Guan WJ, Ni ZY, Hu Y, Liang WH, Ou CQ, $\mathrm{He} \mathrm{JX}$, et al. Clinical characteristics of coronavirus disease 2019 in China. N Engl J Med. 2020;382(18):1708-20.

2 Huang C, Wang Y, Li X, Ren L, Zhao J, Hu Y, et al. Clinical features of patients infected with 2019 novel coronavirus in Wuhan, China. Lancet. 2020;395:497-506.

3 Cheng Y, Luo R, Wang K, Zhang M, Wang Z, Dong $\mathrm{L}$, et al. Kidney disease is associated with in-hospital death of patients with COVID-19. Kidney Int. 2020 May;97(5):829-38.

4 Naicker S, Yang CW, Hwang SJ, Liu BC, Chen JH, Jha V. The novel coronavirus 2019 epidemic and kidneys. Kidney Int. 2020 May; 97(5):824-8.

5 Petrilli CM, Jones SA, Yang J, Rajagopalan H, O'Donnell L, Chernyak Y, et al. Factors associated with hospital admission and critical illness among 5279 people with coronavirus disease 2019 in New York city: prospective cohort study. BMJ. 2020;369:m1966.

6 Williamson EJ, Walker AJ, Bhaskaran K, Bacon S, Bates C, Morton CE, et al. Factors associated with COVID-19-related death using OpenSAFELY. Nature. 2020 Aug;584(7821): 430-6.

7 Gupta A, Madhavan MV, Sehgal K, Nair N, Mahajan S, Sehrawat TS, et al. Extrapulmonary manifestations of COVID-19. Nat Med. 2020;26(7):1017-32.

8 Chan L, Chaudhary K, Saha A, Chauhan K, Vaid A, Zhao S, et al. AKI in hospitalized patients with COVID-19. J Am Soc Nephrol. 2020;32(1):151-60.

$9 \mathrm{Ng} \mathrm{JH}$, Hirsch JS, Hazzan A, Wanchoo R, Shah HH, Malieckal DA, et al. Northwell ne- phrology COVID-19 research consortium members. outcomes among patients hospitalized with COVID-19 and acute kidney injury. Am J Kidney Dis. 2020 Sep;30998-6386: S0272-7.

10 Nikolopoulou A, Teixeira C, Cook HT, Roufosse C, Cairns THD, Levy JB, et al. Membranous nephropathy associated with viral infection. Clin Kidney J. 2020.

11 Söderholm J, Millbourn C, Büsch K, Kövamees J, Schvarcz R, Lindahl K, et al. Higher risk of renal disease in chronic hepatitis $\mathrm{C} \mathrm{pa-}$ tients: antiviral therapy survival benefit in patients on hemodialysis. J Hepatol. 2018 May; 68(5):904-11.

12 Peleg Y, Kudose S, D'Agati V, Siddall E, Ahmad S, Nickolas T, et al. Acute kidney injury due to collapsing glomerulopathy following COVID-19 infection. Kidney Int Rep. 2020 Apr 28;5(6):940-5.

13 Varga Z, Flammer AJ, Steiger P, Haberecker $\mathrm{M}$, Andermatt R, Zinkernagel AS, et al. Endothelial cell infection and endotheliitis in COVID-19. Lancet. 2020 Apr 20;395(10234): 1417-8.

14 Spyropoulos AC, Ageno W, Barnathan ES. Hospital-based use of thromboprophylaxis in patients with COVID-19. Lancet. 2020 May 2; 395(10234):e75.

15 https: //www.folkhalsomyndigheten.se/globalassets/statistik-uppfoljning/smittsammasjukdomar/veckorapporter-covid-19/2020/ covid-19-veckorapport-vecka-31-final.pdf.

16 Alberici F, Delbarba E, Manenti C, Econimo L, Valerio F, Pola A, et al. Management of patients on dialysis and with kidney transplant during SARS-COV-2 (COVID-19) pandemic in Brescia, Italy [published online ahead of print, 2020 Apr 4]. Kidney Int Rep. 2020;5(5): 580-5.

17 Creput C, Fumeron C, Toledano D, Diaconita M, Izzedine H. COVID-19 in patients undergoing hemodialysis: prevalence and asymptomatic screening during a period of high community prevalence in a large paris center. Kidney Med. Nov-Dec 2020;2(6):716-23.e1.

$18 \mathrm{https} / / / \mathrm{www} \cdot$ revistanefrologia.com/en-status-sars-cov-2-infection-in-patients-avanceS201325142030050X

19 Peter H, Mafham M, Linsell L, Bell JL, Staplin $\mathrm{N}$, Emberson JR, et al. Effect of hydroxychloroquine in hospitalized patients with $\mathrm{Cov}$ id-19: preliminary results from a multi-centre, randomized, controlled trial. MedRxiv. 2020;07:20151852.

20 ERA-CODA ERA-EDTA, presented at the ERA-EDTA virtual congress June 6-9, 2020.

21 Jager KJ, Kramer A, Chesnaye NC, Couchoud C, Sánchez-Álvarez JE, Garneata L, et al. Results from the ERA-EDTA registry indicate a high mortality due to COVID-19 in dialysis patients and kidney transplant recipients across Europe. Kidney Int. 2020 Oct 15;98(6): 1540-8.

22 Sachdeva M, Uppal NN, Hirsch JS, Ng JH, Malieckal D, Fishbane S, et al. COVID-19 in hospitalized patients on chronic peritoneal dialysis: a case series. Am J Nephrol. 2020; 51(8):669-74.

23 Ma Y, Diao B, Lv X, Zhu J, Chen C, Liu L, et al. Epidemiological, clinical, and immunological features of a cluster of COVID-19-contracted hemodialysis patients. Kidney Int Rep. 2020;5(8):1333-41.
COVID-19 in Hemodialysis and Peritoneal Dialysis Patients in Sweden 
24 Clarke C, Prendecki M, Dhutia A, Ali MA, Sajjad H, Shivakumar O, et al. High prevalence of asymptomatic COVID-19 infection in hemodialysis patients detected using serologic screening. J Am Soc Nephrol. 2020 Sep;31(9): 1969-75.

25 Chan M, Ostermann M. Outcomes of chronic hemodialysis patients in the intensive care unit. Crit Care Res Pract. 2013;2013:715807.

26 Sparks MA, South A, Welling P, Luther JM, Cohen J, Byrd JB, et al. Sound science before quick judgement regarding RAS blockade in COVID-19. Clin J Am Soc Nephrol. 2020; 15(5):714-6.

27 Lopes RD, Macedo AVS, de Barros E Silva PGM, Moll-Bernardes RJ, Feldman A, D'Andréa Saba Arruda G, et al. Continuing versus suspending ACE inhibitors and ARBs: impact of adverse outcomes in hospitalized patients with Covid-19: the BRACE CORONA trial. Am Heart J. 2020 Aug;226:49-59.

28 Bean D, Kraljevic Z, Searle T, Bendayan R, O'Gallaghe K, Pickles A, et al. Treatment with
ACE-inhibitors is associated with less severe disease with SARS-Covid-19 infection in a multi-site UK acute Hospital Trust. MedRxiv. 2020.

29 Chawki S, Buchard A, Sakhi H, Dardim K, Sakhawi KE, Chawki M, et al. Treatment Impact on COVID-19 evolution in hemodialysis patients. Kidney Int. 2020;98(4):1053-54.

$30 \mathrm{https} / /$ www.medscinet.net/snr/rapporterdocs / Svenskt \% $20 \mathrm{Njurregister \% 20}$ A\%CC\%8Arsrapport\%202019.pdf.

31 Klopfenstein T, Zayet S, Lohse A, Balblanc JC, Badie J, Royer PY, et al. Tocilizumab therapy reduced intensive care unit admissions and/ or mortality in COVID-19 patients. Med Mal Infect. 2020;50(5):397-400.

32 Fadel R, Morrison AR, Vahia A, Smith ZR, Chaudhry Z, Bhargava P, et al. Early short course corticosteroids in hospitalized patients with COVID-19. Clin Infect Dis. 2020 Nov 19;71(16):2114-20

33 RECOVERY Collaborative Group; Horby P, Lim WS, Emberson JR, Mafham M, Bell JL, et al. Dexamethasone in hospitalized patients with Covid-19: preliminary report. N Engl J Med. 2020 Jul 17:NEJMoa2021436.

34 Puntmann VO, Carerj ML, Wieters I, Fahim M, Arendt C, Hoffmann J, et al. Outcomes of cardiovascular magnetic resonance imaging in patients recently recovered from coronavirus disease 2019 (COVID-19). JAMA Cardiol. 2020 Nov 1;5(11):1265-73.

35 Greenhalgh T, Knight M, A'Court C, Buxton M, Husain L. Management of post-acute covid-19 in primary care. BMJ. 2020 Aug 11; 370:m3026.

36 Zoccali C, Blankestijn PJ, Bruchfeld A, Capasso G, Fliser D, Fouque D, et al. Children of a lesser god: exclusion of chronic kidney disease patients from clinical trials. Nephrol Dial Transplant. 2019 Jul 1;34(7):1112-4.

37 Adamsick ML, Gandhi RG, Bidell MR, Elshaboury RH, Bhattacharyya RP, Kim AY, et al. Remdesivir in patients with acute or chronic kidney disease and COVID-19. J Am Soc Nephrol. 2020 Jul;31(7):1384-6. 